\title{
Implantação de Serviços no Âmbito do Cuidado Farmacêutico em uma Central de Distribuição de Medicamentos
}

\section{Implementation of Services In The Framework of Pharmaceutical Care in a Drug Distribution Center}

\section{Implementación de Servicios en el Marco de la Atención Farmacéutica en un Centro de Distribución de Medicamentos}

\footnotetext{
(iD) Aline Karina Maure

Universidade Federal de Alfenas (UNIFAL), Alfenas, Minas Gerais, Brasil alinemaure@hotmail.com
}

\section{iD) (9iliana Batista Vieira \\ Universidade Federal de Alfenas (UNIFAL), Alfenas, Minas Gerais, Brasil lilianabvieira@yahoo.com.br}

\section{(D) Ricardo Radighieri Rascado Universidade Federal de Alfenas (UNIFAL), Alfenas, Minas Gerais, Brasil ricardounifal@gmail.com \\ (D) Universidade Federal de Alfenas (UNIFAL), Alfenas, Minas Gerais, Brasil tiagomarques farmacia@yahoo.com.br}

\section{0}

Luciene Alves Moreira Marques

Universidade Federal de Alfenas (UNIFAL), Alfenas, Minas Gerais, Brasil luciene.marques@unifal-mg.edu.br

\begin{abstract}
Resumo: O Cuidado Farmacêutico foi implantado em vários cenários nas últimas décadas. Este estudo teve como objetivo descrever a implantação do Cuidado Farmacêutico na atenção básica de saúde de um município de médio porte do sul de Minas Gerais, Brasil. Este é um relato de experiência de um trabalho desenvolvido entre novembro
\end{abstract}


de 2017 e setembro de 2018. Os serviços ofertados foram definidos em parceria entre universidade e gestor da Assistência Farmacêutica Municipal. O gestor forneceu o espaço físico e mobiliário e a universidade forneceu os equipamentos e mão de obra qualificada. Após doze meses de implantação, foi possível identificar fatores facilitadores e barreiras no processo, o que poderá ser utilizado como experiência para expandir o serviço a outros estabelecimentos de saúde do município e servir de modelo para a implantação do Cuidado Farmacêutico em outros cenários.

Palavras-chave: Cuidado Farmacêutico. Atenção Farmacêutica. Serviços farmacêuticos.

Abstract: Pharmaceutical Care has been implemented in several scenarios in the last decades. This study aimed to describe the implementation of Pharmaceutical Care in primary health care in a medium-sized municipality in southern Minas Gerais, Brazil. This is an experience report of a work developed between November / 2017 and September / 2018. The services offered were defined in partnership between the university and the municipal pharmaceutical assistance manager. The manager provided the physical space and furniture and the university provided the equipment and qualified labor. After twelve months of implementation, it was possible to identify facilitating factors and barriers in the process, which can be used as an experience to expand the service to other health establishments in the municipality and serve as a model for the implementation of Pharmaceutical Care in other scenarios.

Keywords: Pharmaceutical care. Pharmaceutical services.

Resumen: Atención farmacéutica se ha implementado en varios escenarios en las últimas décadas. Este estudio tuvo como objetivo describir la implementación de la Atención Farmacéutica en la atención primaria de salud en un municipio de tamaño mediano en el sur de Minas Gerais, Brasil. Se trata de un relato de experiencia de un trabajo 
desarrollado entre noviembre de 2017 y septiembre de 2018. Los servicios ofrecidos fueron definidos en alianza entre la universidad y el gerente de la Asistencia Farmacéutica Municipal. El gerente proporcionó el espacio físico y el mobiliario y la universidad proporcionó el equipo y la mano de obra calificada. Luego de doce meses de implementación, se logró identificar factores facilitadores y barreras en el proceso, los cuales pueden ser utilizados como experiencia para expandir el servicio a otros establecimientos de salud del municipio y servir de modelo para la implementación de la Atención Farmacéutica en otros escenarios.

Palabras clave: Atención farmacêutica. Servicios farmacéuticos.

Data de submissão: 29/09/2020

Data de aprovação: 16/12/2020 
Implantação de Serviços no Âmbito do Cuidado Farmacêutico em uma Central de... Aline Karina Maure • Liliana Batista Vieira $\bullet$ et al..

\section{Introdução}

Hepler e Strand (1990) apontaram os problemas relacionados aos medicamentos (PRM) como causas de morbimortalidade e um grande problema de saúde pública não apenas nos Estados Unidos, mas em todo o mundo. Esses problemas podem ter causas diversas, decorrentes de falhas na necessidade, efetividade, segurança ou conveniência ao tratamento (BASGER; MOLES; CHEN, 2014). Estes problemas geram impactos negativos na resposta clínica dos pacientes, e também podem gerar impactos econômicos e na qualidade de vida dos mesmos. O Cuidado Farmacêutico é definido como:

Um modelo de prática que orienta a provisão de diferentes serviços farmacêuticos diretamente destinados ao paciente, à família e à comunidade, visando à prevenção e resolução de problemas da farmacoterapia, ao uso racional e ótimo dos medicamentos, à promoção, à proteção e à recuperação da saúde, bem como à prevenção de doenças e de outros problemas de saúde (CONSELHO FEDERAL DE FARMÁCIA, 2013; 2016).

O Cuidado Farmacêutico através de intervenções no processo de uso dos medicamentos contribui para uma farmacoterapia racional e a minimização de impactos econômicos (ARAÚjO et al., 2017).

O farmacêutico pode desempenhar um conjunto de ações denominadas serviços clínicos que tem por objetivo oferecer atenção integral e humanizada, tendo o medicamento como componente essencial e buscando o 
Implantação de Serviços no Âmbito do Cuidado Farmacêutico em uma Central de... Aline Karina Maure • Liliana Batista Vieira • et al .

uso racional do mesmo. Tais ações desenvolvidas por um farmacêutico, ou sob sua coordenação, incorporadas a uma equipe de saúde e com a participação comunitária, têm como objetivo a obtenção de resultados concretos em saúde que visam à melhoria da qualidade de vida do indivíduo, da família e da comunidade (ORGANIZACION PANAMERICANA DE LA SALUD, 2013; CONSELHO FEDERAL DE FARMÁCIA, 2016).

O Programa de Suporte ao Cuidado Farmacêutico na Atenção à Saúde, denominado PROFAR, enumera nove serviços farmacêuticos clínicos: dispensação, rastreamento em saúde, manejo de problemas de saúde autolimitados ou transtornos menores, educação em saúde, revisão da farmacoterapia, monitorização terapêutica, conciliação de medicamentos, seguimento farmacoterapêutico e gestão da condição de saúde (CONSELHO FEDERAL DE FARMÁCIA, 2016).

O aumento da expectativa de vida, teve como consequência o aumento de doenças crônicas não transmissíveis e de seus fatores de risco (NASRI, 2008; CAMARGOS; GONZAGA, 2015). As doenças crônicas remetem normalmente a comorbidades que necessitam do uso de medicamentos continuamente para a melhora do quadro de saúde do indivíduo. Portanto, os serviços clínicos realizados por farmacêuticos são estratégias importantes e necessárias diante desse momento histórico para garantir o sucesso do tratamento medicamentoso, o que acontece 
Implantação de Serviços no Âmbito do Cuidado Farmacêutico em uma Central de... Aline Karina Maure • Liliana Batista Vieira • et al _

quando os itens prescritos são necessários (indicação), efetivos, seguros e convenientes (adesão).

Diante disso, o objetivo deste artigo é relatar a experiência de implantação de serviços clínicos realizados por farmacêuticos em uma unidade pública de saúde, descrevendo os pontos facilitadores e barreiras desse processo.

\section{Métodos}

Trata-se de um relato de experiência referente à implantação de serviços clínicos realizados por farmacêuticos em uma unidade pública de saúde em um município do sul de Minas Gerais. O município em que o estudo foi realizado possui, atualmente, 77.618 habitantes, segundo estimativa do IBGE (Instituto Brasileiro de Geografia e Estatística), e 12 Programas de Saúde da Família. Possui ainda duas Instituições de Ensino Superior (IES), uma pública e outra particular, nas quais há o curso de Farmácia. Conta com três farmacêuticos no quadro de recursos humanos, sendo um alocado na Central de Distribuição de Medicamentos (CDM) e os outros dois no Centro de Atenção Psicossocial e no Centro de Testagem e aconselhamento.

A implantação da proposta envolveu um Planejamento Estratégico Situacional (PES), estruturado à luz das teorias de gestão de Matus (1993). Pelo PES, o processo de gestão envolve a interdependência entre três dimensões: projeto de governo, governabilidade e capacidade de governo. 
Implantação de Serviços no Âmbito do Cuidado Farmacêutico em uma Central de... Aline Karina Maure • Liliana Batista Vieira • et al _

Nesse contexto, o projeto de governo se estabeleceu por meio de um projeto de Extensão Universitária, registrado pela Pró-Reitoria de Extensão da IES pública sediada no município. A governabilidade, entendida como construção de fortes alianças para reduzir a resistência ao projeto de governo, consolidou-se pela parceria firmada com a Secretaria Municipal de Saúde (SMS) e Coordenadoria de Assistência Farmacêutica. Por sua vez, a capacidade de governo, que diz respeito à importância de manter e mobilizar recursos operacionais, técnicos e humanos para a consecução do projeto, consolidou-se pelo envolvimento de docentes e discentes do curso de Farmácia da IES no desenvolvimento e execução do processo de implantação.

O local definido para a implantação foi um estabelecimento de saúde pública, vinculado à atenção primária. Esse estabelecimento, denominado Central de Distribuição de Medicamentos (CDM), caracteriza-se como um local de simples fornecimento de medicamentos. Assim sendo, a implantação dos serviços clínicos no estabelecimento visava à sua ressignificação, contemplando a promoção, proteção e prevenção em saúde para, dessa forma, ser possível contribuir na racionalização dos limitados recursos financeiros do SUS e do uso dos medicamentos distribuídos no local. Por isso, três serviços clínicos no âmbito do Cuidado Farmacêutico foram estabelecidos: acompanhamento farmacoterapêutico, educação em saúde e rastreamento em saúde. A escolha desses serviços se justificou pelas condições estruturais do 
Implantação de Serviços no Âmbito do Cuidado Farmacêutico em uma Central de... Aline Karina Maure • Liliana Batista Vieira • et al _

local, pelo tipo de medicamento fornecido, pela disponibilidade dos recursos necessários e pelo perfil de demandas em saúde do público atendido.

Pela pactuação entre as partes, a SMS disponibilizou o espaço físico e mobiliário, enquanto a contrapartida da IES foram os instrumentos e recursos humanos qualificados para funcionamento dos serviços.

Após a aprovação do projeto e estruturação das condições para a oferta dos serviços, a partir da governabilidade e capacidade de governo, os serviços clínicos no âmbito do Cuidado Farmacêutico passaram a ser divulgados junto à equipe multiprofissional de saúde que atua nas Unidades Básicas de Saúde (UBS) do município e comunidades usuárias desses estabelecimentos. Para isso, utilizou-se como estratégia o envio de carta aos profissionais de saúde e apresentação, por meio da projeção de slides, para a população nas salas de espera de cada UBS. Estratégias adicionais como distribuição de panfleto explicativo para a população durante as campanhas de saúde, realizadas pela IES em praça pública, e elaboração de páginas nas redes sociais (Facebook, Instagram e um blog) também foram utilizadas para a divulgação do Cuidado Farmacêutico. Em adição, foram também concedidas entrevistas na rádio local para divulgar os serviços farmacêuticos.

A partir dessa divulgação e da discussão com os atores envolvidos (usuários do SUS, profissionais de saúde, gestores das UBS, docentes e discentes), definiu-se que 
Implantação de Serviços no Âmbito do Cuidado Farmacêutico em uma Central de... Aline Karina Maure • Liliana Batista Vieira • et al _

seriam ofertados os seguintes serviços: educação em saúde, rastreamento em saúde e acompanhamento farmacoterapêutico. Essa decisão, estabelecida de forma democrática, teve como embasamento as demandas em saúde da comunidade e os possíveis resultados que poderiam ser obtidos de cada serviço, considerando suas peculiaridades procedimentais.

Os serviços implantados na CDM iniciaram com a oferta por quatro docentes da IES e discentes estagiários e extensionistas que se revezavam no atendimento à população. Pelo fato de haver na CDM um único farmacêutico do quadro de recursos humanos, o qual se responsabiliza pelas atividades técnico-gerenciais desta e de outras treze farmácias estabelecidas em unidades de saúde do município, o mesmo apoiou a implantação dos serviços, mas não pôde envolver-se diretamente na realização dos mesmos.

A capacitação dos discentes envolvidos no processo, que já possuíam conhecimento teórico inicial pelo cumprimento de disciplinas da graduação relacionadas ao Cuidado Farmacêutico, aconteceu em serviço, pois em todos os horários de funcionamento esteve presente um docente responsável. Dessa forma, o treinamento se apoiou no modelo conceitual formativo proposto por Miller (1990), segundo o qual a competência para a atuação clínica advém da formação de bases cognitivas ("saber" e "saber como fazer") da prática profissional ("fazer") e a necessidade da 
Implantação de Serviços no Âmbito do Cuidado Farmacêutico em uma Central de... Aline Karina Maure • Liliana Batista Vieira • et al _

avaliação de habilidades e competências práticas ("mostrar como faz").

O público alvo dos serviços foram pessoas de ambos os sexos, sem restrição de idade, em uso de medicamentos (preferencialmente polimedicados), com suspeitas de problemas na farmacoterapia ou dúvidas sobre medicamentos. A abordagem dos sujeitos acontecia na própria CDM (busca ativa), após a retirada do medicamento pelo paciente, pelo encaminhamento pelos atendentes que trabalhavam no fornecimento dos medicamentos ou pelo encaminhamento por médicos das UBS.

\section{Resultados e Discussão}

No período de novembro de 2017 a setembro de 2018 foram atendidos 75 pacientes no serviço de acompanhamento farmacoterapêutico e 220 pacientes nos serviços de educação em saúde e o rastreamento em saúde. A busca ativa foi a forma de abordagem que motivou praticamente todos os atendimentos, sendo que dois pacientes foram encaminhados à equipe do Cuidado Farmacêutico por médicos. O farmacêutico muitas vezes não é reconhecido como membro da equipe de saúde e sua presença regular não é percebida como necessária. Isso se deve ao contexto histórico de pouca inserção em atividades de atenção básica e de falta de reconhecimento social, além de formação técnica com pouca ênfase na parte clínica (LOCH-NECKEL; CREPALDI, 2009). Além disso, o modelo de 
Implantação de Serviços no Âmbito do Cuidado Farmacêutico em uma Central de... Aline Karina Maure • Liliana Batista Vieira • et al _

trabalho multiprofissional existente pressupõe níveis de subordinação, o que gera insatisfações na equipe e interfere na integralidade do cuidado ao indivíduo (MACEDO, 2007).

Cada consulta de acompanhamento farmacoterapêutico durava em média 60 minutos e os retornos, 30 minutos. A periodicidade de agendamento dependia de cada caso clínico. No entanto, a média era de 30 dias para o retorno.

O serviço de rastreamento em saúde, por sua vez, durava 15 minutos. Neste serviço, eram executados os procedimentos de aferição da pressão arterial e determinação da glicemia capilar. Caso o paciente apresentasse algum parâmetro alterado, era convidado a participar do acompanhamento farmacoterapêutico ou encaminhado a outro profissional de saúde.

A educação em saúde, como serviço isolado do acompanhamento farmacoterapêutico e do rastreamento em saúde, era realizada quando o paciente solicitava algum esclarecimento sobre o seu tratamento no balcão de atendimento. O processo durava de 15 a 30 minutos, dependendo do assunto abordado. No processo de educação em saúde eram usados recursos como fornecimento de moldes para facilitar a localização e rodízio da aplicação de insulina, caixas para organizar medicamentos de pacientes em polifarmácia e caderno de receitas saudáveis de forma a auxiliá-los em uma dieta balanceada. No estudo realizado por Araújo et al. (2017) foi observado pouca participação dos farmacêuticos 
Implantação de Serviços no Âmbito do Cuidado Farmacêutico em uma Central de... Aline Karina Maure • Liliana Batista Vieira • et al _

entrevistados em atividades educativas na atenção básica.

Talvez isso seja um dos motivos para a utilização inadequada dos medicamentos, pouca compreensão sobre a doença e não adesão ao tratamento por parte de alguns pacientes.

Durante a implantação dos serviços clínicos, foi possível observar e identificar variantes/interferentes que outros estudos não obtiveram. A literatura mostra que a implantação desses serviços geralmente acontece em hospitais e UBS (OBRELI-NETO, 2015; BERNARDI; RODRIGUES; TOMPOROSKI, 2014), nos quais existe uma série de profissionais atuando no mesmo local, o que possibilita um atendimento multiprofissional de forma a somar e apoiar os serviços farmacêuticos. No caso da CDM, a falta de um apoio multiprofissional no local de atendimento e a indisponibilidade dos prontuários, que ficam arquivados em meio físico nas UBS, diminuiu a agilidade na resolução de problemas relacionados aos medicamentos (PRM), dificultando a elaboração de intervenções e análise de resultados, pois os farmacêuticos necessitavam deslocar-se até à UBS para consultar os prontuários quando necessário. Contudo, isso não impossibilitou a prática do Cuidado Farmacêutico.

Uma outra dificuldade encontrada foi o horário restrito de atendimento para os pacientes, realizados das $13 \mathrm{~h}$ às $17 \mathrm{~h}$ horas, pois a sala disponibilizada foi dividida com as atividades da Assistência Social, que funciona na parte da manhã. Isso limitou o atendimento, pois alguns pacientes 
Implantação de Serviços no Âmbito do Cuidado Farmacêutico em uma Central de... Aline Karina Maure • Liliana Batista Vieira • et al _

que trabalham à tarde, e só poderiam consultar pela manhã, não puderam ser contemplados.

O sistema público não possui farmacêuticos em número suficiente para atender a demanda do município e, consequentemente, não há um farmacêutico disponível para realizar as atividades clínicas, limitando o Cuidado Farmacêutico aos pacientes ao calendário letivo da universidade. De acordo com Araújo et al. (2017), as atividades clínicas são desenvolvidas, em diversos países, em regime de dedicação integral pelo farmacêutico não havendo sobreposição de atividades técnico-gerenciais e atividades clínicas. Até porque os farmacêuticos que executam os serviços clínicos não são autorizados por lei a exercer outras atividades.

Um fator facilitador foi o apoio recebido pela Comissão de Farmácia e Terapêutica do município, que atualizou um guia sobre dispensação de medicamentos vigente. Nesse documento, elaborado anteriormente pela Secretaria Municipal de Saúde, foi incluído o farmacêutico como prescritor e solicitante de exames laboratoriais, conforme determina as Resoluções do Conselho Federal de Farmácia $n^{\circ} 585 / 13$ e n 586/13. A versão atualizada do documento foi sancionada e publicada por decreto municipal.

Espera-se ampliar a oferta dos serviços para as UBS do município. No entanto, o fator limitante é a deficiência de mão de obra qualificada para tal, ou seja, a presença do farmacêutico nas unidades. 
Implantação de Serviços no Âmbito do Cuidado Farmacêutico em uma Central de... Aline Karina Maure $\cdot$ Liliana Batista Vieira $\bullet$ et al _

Nesse sentido, as atividades desenvolvidas no consultório farmacêutico foram apresentadas para a Câmara Municipal de Vereadores, com o objetivo de sensibilizá-los da necessidade de um profissional farmacêutico exclusivo para desenvolver as atividades clínicas no município.

\section{Conclusões}

A implantação de serviços clínicos realizados por farmacêuticos na unidade saúde do município do estudo possibilitou a consolidação do Cuidado Farmacêutico na rede pública de saúde. Permitiu a concretização dos aprendizados teóricos pelos estudantes envolvidos na ação em associação com a vivência da prática clínica, favorecendo a relação ensino-serviço-comunidade e a busca pelo uso racional de medicamentos.

Identificou-se como barreiras para a implantação do serviço 0 excesso de atividades administrativas desempenhadas pelos farmacêuticos, escassez de recursos humanos, falta de acesso aos medicamentos pela população e sensibilização dos gestores públicos para a importância desta prática. Todavia, o apoio de entidades como a Comissão de Farmácia e Terapêutica e do poder legislativo municipal foram facilitadores que contribuíram para a governabilidade e capacidade de governo da ação, permitindo a execução do projeto de implantação e o início da oferta dos serviços no município. 
Implantação de Serviços no Âmbito do Cuidado Farmacêutico em uma Central de... Aline Karina Maure • Liliana Batista Vieira • et al..

Acredita-se que essas experiências permitirão expandir os serviços a outros estabelecimentos de saúde do município e favorecer a inserção do farmacêutico na equipe de saúde. Além disso, este relato do percurso metodológico do processo de implantação do Cuidado Farmacêutico poderá servir de modelo para que resultados semelhantes sejam possíveis também em outros cenários.

\section{Referências}

ARAúJO, P. S. et al. Atividades farmacêuticas de natureza clínica na atenção básica no Brasil. Revista Saúde Pública, v. 51, 2017. Supl 2:6s. BASGER, B.J.; MOLES, R.J.; CHEN, T.F. Application OF DRUG-RELATED PROBLEM (DRP) CLASSIFICATION SYSTEMS: A REVIEW OF THE LITERATURE. EUR J Clin Pharmacol, v. 70, 2014, P. 799-815.

BERNARDI, E. A. T.; RODRIGUES, R.; TOMPOROSKI, G. G. IMPLANTAÇÃO DA AVALIAÇÃO FARMACÊUTICA DA PRESCRIÇÃO MÉDICA E AS AÇÕES DE FARMÁCIA CLÍNICA EM UM HOSPITAL ONCOLÓGICO DO SUL DO BRASIL.

Revista Espaço para a Saúde, Londrina, v. 15, N. 2, jun 2014, P. 29-36.

CAMARGOS, M. C. S.; GONZAGA, M. R. ViVER MAIS E MELHOR?

ESTIMATIVAS DE EXPECTATIVA DE VIDA SAUDÁVEL PARA A POPULAÇÃO BRASILEIRA. Cadernos de Saúde Pública, v. 31, 2015, P. 1460-1472.

CONSELHO FEDERAL DE FARMÁCIA (CFF). REsolução №. 585, de 29 de Agosto de 2013. Regulamenta as ATRIBUiçõEs CLínICAS do FARMACÊUTICO E dÁ OUTRAS PROVIDÊNCIAS. DIÁRIO OfICIAL DA UNIÃo, PODER Executivo, Brasília, DF, 25 set. 2013. Seção 1, p. 186-188.

CONSELHO FEDERAL DE FARMÁCIA (CFF). ServiçOS FarmacêutıCos DIRETAMENTE DESTINADOS AO PACIENTE, À FAMílla E À COMUNIDADE: CONTEXtualização e arcabouço conceitual/Conselho Federal de Farmácia. - Brasília: Conselho Federal de Farmácia, 2016. HEPLER, C.D.; STRAND, L.M. OPPORTUNITIES AND RESPONSIBILITIES IN pharmaceutical Care. Am J Hosp Pharm, v. 47, 1990, p. 533-543. 
Implantação de Serviços no Âmbito do Cuidado Farmacêutico em uma Central de... Aline Karina Maure • Liliana Batista Vieira • et al.

LOCH-NECKEL, G.; CREPALDI, M.A. PhARMACIST CONTRIBUtIONS FOR BASIC CARE FROM THE PERSPECTIVE OF PROFESSIONALS OF FAMILIAL HEALTH CARE teams. Brazilian J Pharm ScI, v. 45, N. 2, 2009, P. 263-272.

LUFT, C.R. O Cuidado Farmacêutico como parte Integrante dos serviços farmacêuticos no Sistema Único de Saúde. 2015.

MACEDO, P.C.M. Desafios atuais no tRabalho MULTIPROFISSIONAL EM SAÚdE. Revista da SBPH, v. 10, N. 2, 2007, P. 33-41.

MATUS, C. Política, planejamento e governo. Brasília: Instituto de Pesquisa Econômica Aplicada, 1993.

MILLER, G. E. The ASSESSMENT OF CLINICAL Skills/competence/performance. Academic Medicine, v. 65, N. 9, 1990, P. 63-67.

NASRI, F. O enVelhecimento populacional no Brasil. Einstein, v. 6, SUPL 1, 2008, P. 4-6.

OBREli-neto, P.R. et.al. Economic Evaluation of a Pharmaceutical Care Program for Elderly Diabetic and Hypertensive Patients in Primary Health Care:A 36-Month Randomized Controlled Clinical. Journal of Managed Care \& Specialty Pharmacy JMCP, v. 21, N. 1, 2015.

ORGANIZACION PANAMERICANA DE LA SALUD (OPS). Servicios FARMACÉUTICOS BASADOS EN LA ATENCIÓN PRIMARIA DE SALUD. DOCUMENTO DE POSICIÓN DE LA OPS/OMS. WASHINGTON, DC: OPS, 2013. 